\title{
In vitro Neuromuscular Junction Induced from Human Induced Pluripotent Stem Cells
}

\author{
Chuang-Yu Lin ${ }^{1}$, Michiko Yoshida ${ }^{1,2}$, Li-Tzu Li $^{3}$, Megumu K. Saito ${ }^{1}$ \\ ${ }^{1}$ Department of Clinical Application, Center for iPS Cell Research and Application (CiRA), Kyoto University ${ }^{2}$ Department of Pediatrics, Kyoto Prefectural \\ University of Medicine ${ }^{3}$ Graduate Institute of Clinical Medicine, Taipei Medical University
}

\section{Corresponding Authors}

Chuang-Yu Lin

chuangyuck@gmail.com

Megumu K. Saito

msaito@cira.kyoto-u.ac.jp

\section{Citation}

Lin, C.Y., Yoshida, M., Li, L.T.,

Saito, M.K. In vitro Neuromuscular Junction Induced from Human Induced

Pluripotent Stem Cells. J. Vis. Exp.

(166), e61396, doi:10.3791/61396 (2020).

\section{Date Published}

December 3, 2020

DOI

$10.3791 / 61396$

URL

jove.com/video/61396

\section{Abstract}

The neuromuscular junction (NMJ) is a specialized synapse that transmits action potentials from the motor neuron to skeletal muscle for mechanical movement. The architecture of the NMJ structure influences the functions of the neuron, the muscle and the mutual interaction. Previous studies have reported many strategies by coculturing the motor neurons and myotubes to generate NMJ in vitro with complex induction process and long culture period but have struggled to recapitulate mature NMJ morphology and function. Our in vitro NMJ induction system is constructed by differentiating human IPSC in a single culture dish. By switching the myogenic and neurogenic induction medium for induction, the resulting NMJ contained pre- and postsynaptic components, including motor neurons, skeletal muscle and Schwann cells in the one month culture. The functional assay of NMJ also showed that the myotubes contraction can be triggered by $\mathrm{Ca}^{++}$then inhibited by curare, an acetylcholine receptor $(\mathrm{AChR})$ inhibitor, in which the stimulating signal is transmitted through NMJ. This simple and robust approach successfully derived the complex structure of NMJ with functional connectivity. This in vitro human NMJ, with its integrated structures and function, has promising potential for studying pathological mechanisms and compound screening.

\section{Introduction}

The neuromuscular junction (NMJ) is a specialized synapse that transmits signals from motor neurons to skeletal muscles to control voluntary muscle movement ${ }^{1,2}$. This synapse is composed of pre- and post-synaptic parts. In the presynaptic part, the motor neuron releases acetylcholine (ACh) from synaptic vesicles by exocytosis. ACh is released from synaptic vesicles to cross the synaptic cleft and to bind to $A C h R$ on the post-synaptic part to trigger an action potential for muscle contraction ${ }^{3,4}$. Any malfunction in this delicate structure can cause NMJ diseases, including spinal muscular atrophy (SMA), congenital myasthenic syndromes (CMS), myasthenia gravis $(M G)^{5,6,7}$, etc. These diseases 
greatly damage the quality of patients' lives and unfortunately have no effective treatment approaches due to the lack of understanding the pathological mechanism. In this study, we aimed to generate in vitro human NMJ from human iPSCs for accurate disease modeling and for therapeutic compounds screening.

Previous studies have demonstrated the possibility of generating in vitro NMJ by co-culture strategies. The motor neurons and skeletal myotubes are generated respectively. The two cell types can be generated from human or mouse primary tissue cultures or be induced from stem cells $8,9,10,11$ and then co-cultured for NMJ formation. The further applications include merging the co-cultured NMJ into microfluidic 3D devices and to use optogenetic units for quantifiable functional assays ${ }^{12,13}$. However, these strategies take a long culture period and require struggle to obtain the primary components of the NMJ, either the motor neuron or the skeletal myotube simultaneously. Yet another important component of NMJ, the Schwann cell, cannot be generated in these culture systems. The advanced culture system that contains the myotube, motor neuron and Schwann cell is desirable as it can offer a reliable and robust model for NMJ studies.

Myogenic Differentiation 1 (MYOD1) is a well-known myogenic regulator for myogenesis ${ }^{14}$. The efficient myogenic differentiation method which applied MYOD1 to drive iPSC into myotubes was built in a previous study ${ }^{15}$. Therefore, we induced the myotubes by overexpressing MYOD1 in iPSCs $^{15}$, and high efficiency of myogenic differentiation was shown. Interestingly, neuron cells appeared along with myotubes spontaneously after day 10 . The appearance of neuron cells in myogenic culture has led us to develop the strategy for generating in vitro NMJ in a single dish. Here we provide a strategy to generate NMJs by overexpressing MYOD1 in human iPSCs for myogenic and motor neuron induction in the same culture dish. The motor neurons are induced spontaneously with several neurotrophic factors (GDNF, BDNF, NT3, etc.) ${ }^{8}$, and, in the meanwhile, the Schwann cells can also be induced ${ }^{16,17}$. Through the interactions among the myotubes, the motor neurons and the Schwann cells, the mature NMJ are formed ${ }^{18}$. This method can generate functional NMJ efficiently, enabling the potential study of pathological mechanisms and therapeutic compound screening.

\section{Protocol}

\section{Preparation of extracellular matrix (ECM)-coated plates}

1. Dilute ECM (see Table of Materials) with ice cold 1x PBS to a final concentration of $2 \%$.

1. Add $1 \mathrm{~mL}$ of $\mathrm{ECM}$ to $49 \mathrm{~mL}$ of $1 \mathrm{x}$ PBS in a 50 $\mathrm{mL}$ plastic tube. Mix well by pipetting up and down several times.

2. Place 1 coverslip into each well of a 6 -well plate. Add 1.5 $\mathrm{mL}$ of $2 \% \mathrm{ECM}$ into each well.

3. Incubate the well plate with $2 \%$ ECM for $2 \mathrm{~h}$ at $37^{\circ} \mathrm{C}$.

4. Aspirate ECM from the well plate and store the plate at $4{ }^{\circ} \mathrm{C}$ before use.

\section{Differentiation of iPSCs toward NMJ}

1. Seed $4 \times 10^{5}$ of iPSCs per well on the 6-well plate prepared in section 1. Make sure to seed the cells on the coverslip pre-deposited in the well.

NOTE: In this study, the 201B7 $7^{\mathrm{MYOD}}$ iPS cell line, a gift from Dr. Sakurai's Lab, was used ${ }^{15}$. 
1. Remove the medium from the iPSCs on the $6 \mathrm{~cm}$ culture dish and wash the iPSCs once with 1x PBS.

2. Add $1 \mathrm{~mL}$ of cell detachment solution to the dish and incubate for $10 \min$ at $37^{\circ} \mathrm{C}$.

3. Add $3 \mathrm{~mL}$ of primate embryonic stem (ES) cell medium to the dish and pipette 3 times gently.

4. Collect the supernatant, which contains detached iPSCs, into a $50 \mathrm{~mL}$ plastic tube and centrifuge at $160 \times g$ at $4{ }^{\circ} \mathrm{C}$ for $5 \mathrm{~min}$.

5. Carefully aspirate the supernatant, resuspend the iPSCs in $3 \mathrm{~mL}$ primate ES cell medium with 10 $\mu \mathrm{M} Y 27632$, and count the cell number using a hemocytometer.

6. Dilute the iPSCs with primate ES cell medium and 10 $\mu \mathrm{M}$ Y27632 to a concentration of $2 \times 10^{5}$ cells $/ \mathrm{mL}$. Add $2 \mathrm{~mL}$ of iPSCs on the coverslip pre-deposited in the well that described in section 1.

2. Induction of the iPSCs to NMJ

1. On day $1,24 \mathrm{~h}$ after seeding the iPSCs to the 6-well plate, remove the culture medium and replace it with $2 \mathrm{~mL}$ of fresh primate ES cell medium containing 1 $\mu \mathrm{g} / \mathrm{mL}$ doxycycline to each well.

2. Change the medium with $2 \mathrm{~mL}$ of myogenic differentiation medium (MDM) (Table 1) containing 1 $\mu \mathrm{g} / \mathrm{mL}$ doxycycline (final concentration) to each well. Refresh the medium every day from day 2 to day 10 .

3. From day 11 , switch the medium to $2 \mathrm{~mL}$ of $\mathrm{NMJ}$ medium (Table 1) to each well. Refresh the medium every 3-4 days thereafter until day 30 .

3. Observe the differentiated NMJ by phase inverted microscopy on day 30 . The NMJ can be used for the following analysis.

\section{Immunofluorescence (IF) staining}

1. On day 30 , aspirate the culture medium from the 6well plate. Fix the NMJ culture by adding $2 \mathrm{~mL}$ of $4 \%$ paraformaldehyde to each well for $30 \mathrm{~min}$ at room temperature.

2. Wash the samples 3 times by adding $2 \mathrm{~mL}$ of $1 \times$ PBS to each well (3 min for each wash).

3. Permeabilize the samples with $0.1 \%$ Triton/PBS for 10 $\min$.

4. Repeat step 3.2.

5. Block the samples with $0.5 \%$ BSA for $1 \mathrm{~h}$ at room temperature.

6. Repeat step 3.2.

7. Incubate the samples with the primary antibodies at 4 ${ }^{\circ} \mathrm{C}$ overnight. The dilution of antibodies is as follows: Islet $1(1 \mu \mathrm{g} / \mathrm{mL})$, myosin heavy chain (MYH) (1/300), neurofilaments (NF) $(1 \mu \mathrm{g} / \mathrm{mL}), \mathrm{S}-100$ (1/300), synaptic vesicle protein 2 (SV2) $(1 \mu \mathrm{g} / \mathrm{mL})$, Tuj1 (1/1000).

8. Repeat step 3.2.

9. Incubate the samples with the secondary antibodies at room temperature for $1 \mathrm{~h}$. The concentration of the secondary antibodies used is as follows: anti-mouse $\lg G 488$ conjugated $(0.1 \mu \mathrm{g} / \mathrm{mL})$, anti-rabbit $\lg G 488$ conjugated $(0.1 \mu \mathrm{g} / \mathrm{mL})$.

10. Repeat step 3.2.

11. Incubate the samples with aBTX-647 $(0.5 \mu \mathrm{g} / \mathrm{mL})$ for AChR staining and with DAPI $(1 \mu \mathrm{g} / \mathrm{mL})$ for nucleus staining.

12. Repeat step 3.2. 
13. Pick up the coverslip with a pair of forceps from the 6well plate and mount it in $50 \%$ glycerol/PBS solution on a microscope slide.

\section{Scanning electron microscopy (SEM)}

1. Fix the NMJ culture with $4 \%$ paraformaldehyde and $1 \%$ glutaraldehyde prepared in $0.1 \mathrm{M}$ potassium phosphate buffer at room temperature for $1 \mathrm{~h}$.

2. Wash the samples 3 times by immersing them in $0.1 \mathrm{M}$ potassium phosphate buffer at room temperature (10 $\mathrm{min}$ for each wash).

3. Dehydrate the samples with ascending concentrations of ethanol $(50 \%, 70 \%, 90 \%, 95 \%$ and $100 \%$ twice). Immerse the samples in each concentration of ethanol for $10 \mathrm{~min}$.

4. Dry the samples by a critical point dryer $\left(-30^{\circ} \mathrm{C}, 0.1\right.$ Torr $)$.

5. Coat the samples with $\mathrm{Pt}$ (platinum) ion coater $(30 \mathrm{~mA}$ for $3 \mathrm{~min}$; thickness of $\mathrm{Pt}$ is about $20 \mathrm{~nm}$ ).

6. Observe the samples by SEM at $5 \mathrm{kV}$.

\section{Transmission electron microscopy (TEM)}

1. Use a cell scraper to harvest the tissue from the NMJ culture plate. Shape the tissue into a small pellet ( 3 $\mathrm{mm}^{3}$ ) with a razor blade and glue by gelation to form a gel-wrapped piece. Fix the gel-wrapped piece with $2 \%$ paraformaldehyde and $2 \%$ glutaraldehyde prepared in $0.1 \mathrm{M}$ potassium phosphate buffer at $4{ }^{\circ} \mathrm{C}$ overnight.

2. Wash the samples 3 times by immersing them in $0.1 \mathrm{M}$ potassium phosphate buffer (15 min for each wash) at room temperature.

3. Postfix the samples with $1 \%$ osmium tetroxide prepared in double distilled $\mathrm{H}_{2} \mathrm{O}$ for $1 \mathrm{~h}$ at room temperature.
CAUTION: This step must be done in a chemical hood.

\section{Repeat step 5.2.}

5. Dehydrate the samples with ascending concentrations of ethanol $(50 \%, 70 \%, 90 \%, 95 \%$ and $100 \%$ twice). Immerse the samples in each concentration of ethanol for $10 \mathrm{~min}$.

6. Aspirate the $100 \%$ ethanol. Infiltrate the samples with ascending volume ratios of epoxy resin to $100 \%$ ethanol mixtures (1:3, 1:1 and 3:1). Agitate each mixture gently at $10 \mathrm{rpm}$ for $1 \mathrm{~h}$ at room temperature.

7. Replace the epoxy-ethanol mixture with pure epoxy resin and agitate gently at $10 \mathrm{rpm}$ for $4 \mathrm{~h}$ at room temperature.

8. After $4 \mathrm{~h}$, refresh the epoxy resin with fresh epoxy resin and agitate gently at $10 \mathrm{rpm}$ overnight at room temperature.

9. Embed the samples in embedding capsules with fresh epoxy resin and cure the samples in an oven at $65{ }^{\circ} \mathrm{C}$ overnight.

10. Ultramicrotomy

1. Coarsely trim the sample blocks under a dissecting microscope for the correct orientation.

2. Finely trim the coarsely trimmed blocks with a glass knife on an ultramicrotome to obtain smooth surfaces.

3. Prepare $70 \mathrm{~nm}$ ultrathin sections from the welltrimmed blocks with a diamond knife. Retrieve the ultrathin sections with 200 mesh carbon-formvarcoated copper grids.

4. Stain the ultrathin sections containing grids with saturated uranyl acetate prepared in double distilled $\mathrm{H}_{2} \mathrm{O}$ for 30 min and wash the grids 3 times with double distilled $\mathrm{H}_{2} \mathrm{O}$ (10 min for each wash). 
5. Further, contrast the ultrathin sections with Reynold's lead citrate ${ }^{19}$ (2.5\%) for $5 \mathrm{~min}$ and wash with boiled $\mathrm{H}_{2} \mathrm{O}$ pre-cooled to room temperature 3 times (10 min for each wash). Put several sodium hydroxide pellets around the staining area to prevent $\mathrm{CO}_{2}$ precipitation on the sections.

11. Observe the ultrathin sections by TEM at $70 \mathrm{kV}$.

\section{Muscle contraction and curare treatment}

1. To trigger the myotube contraction, add $25 \mathrm{mM} \mathrm{CaCl}_{2}$ in the culture medium in step 2.3. The movement of myotubes can be observed in 1-2 min.

2. Place the 6-well plate on the stage of an inverted microscope. Record a movie of myotube contraction by live cell microscopy.

3. To stop the myotube contraction, add curare to the culture medium $(300 \mathrm{ng} / \mathrm{mL})$. Then record the movie as step 6.2.

4. Open the movie file with motion vector analysis software then click the Motion Analysis button to analyze the movie.

NOTE: The myotubes contraction is shown as timemotion graphic. The movies are color-coded to show the movement speed of myotubes. The color-coded sparkly signals indicate the movements of myotubes where the red color shows the fastest movement speed and blue color shows the slowest movement speed.

\section{Representative Results}

Using our differentiation strategy, the culture showed preand post-synaptic components of the NMJ on day 30 . The NMJ components were induced and well-developed in a single well, and their morphology and locations in the NMJ were demonstrated by IF microscopy (Figure 1). The flow chart in Figure 1A summarizes the time course of the NMJ differentiation progression. The staining of neurofilaments (NF), synaptic vesicles (SV2) and AChR (Figure 1B,C) indicate the neurons. The single staining images of NF and SV2 are shown in Supplementary Figure 1. The alphabungarotoxin staining indicates $A C h R$ (Figure 1C). The merged image shows the relative locations of a motor neuron and AChR in the NMJ (Figure 1D,E). The second set of IF staining indicates the motor neuron by Tuj 1 and Islet 1 (Figure $1 \mathrm{G}, \mathrm{H})$ and post-synaptic myotubes by myosin heavy chain (Figure 1I). The Schwann cells were also labeled by S-100 antibody in the NMJ culture (Supplementary Figure 2).

To further confirm the identities of the NMJ components, we used SEM for detailed morphological analysis. Mature NMJ morphology with expanded axon terminals, axons and muscle fibers are shown in Figure 2A,B. TEM was performed to reveal the mature ultrastructure of the NMJ components including the pre-synaptic axon terminal with synaptic vesicles and the post-synaptic part, which is separated by the synaptic cleft (Figure 2C-E). Junctional folds are indicated by the yellow dashed-line, which marks the junction of the neuron and muscle fibers (Figure 2C). The mature axon terminals that contain synaptic vesicles are shown in Figure 2D,E (yellow arrows). The morphological results show that the NMJ components were well induced and matured.

To evaluate the function of the in vitro NMJ, we performed motion analysis by stimulating the motor neuron with $\mathrm{CaCl}_{2}$ to trigger myotube contractions. The results showed that $\mathrm{Ca}^{2+}$ can trigger muscle contractions. The contractions could be interrupted by curare. The NMJ showed prominent motion signals that disappeared with curare treatment 
(Figure 3). This effect confirms that the motor neuron signals were transmitted through the NMJ to trigger muscle contraction. Taken together, the IF microscopy, SEM and TEM data demonstrated the spatial distribution, morphology and maturity of the NMJ components in the in vitro NMJ generated by the protocol described above. Motion analysis validated the function of the in vitro NMJ, which implies its potential application to developing therapeutic strategies. 

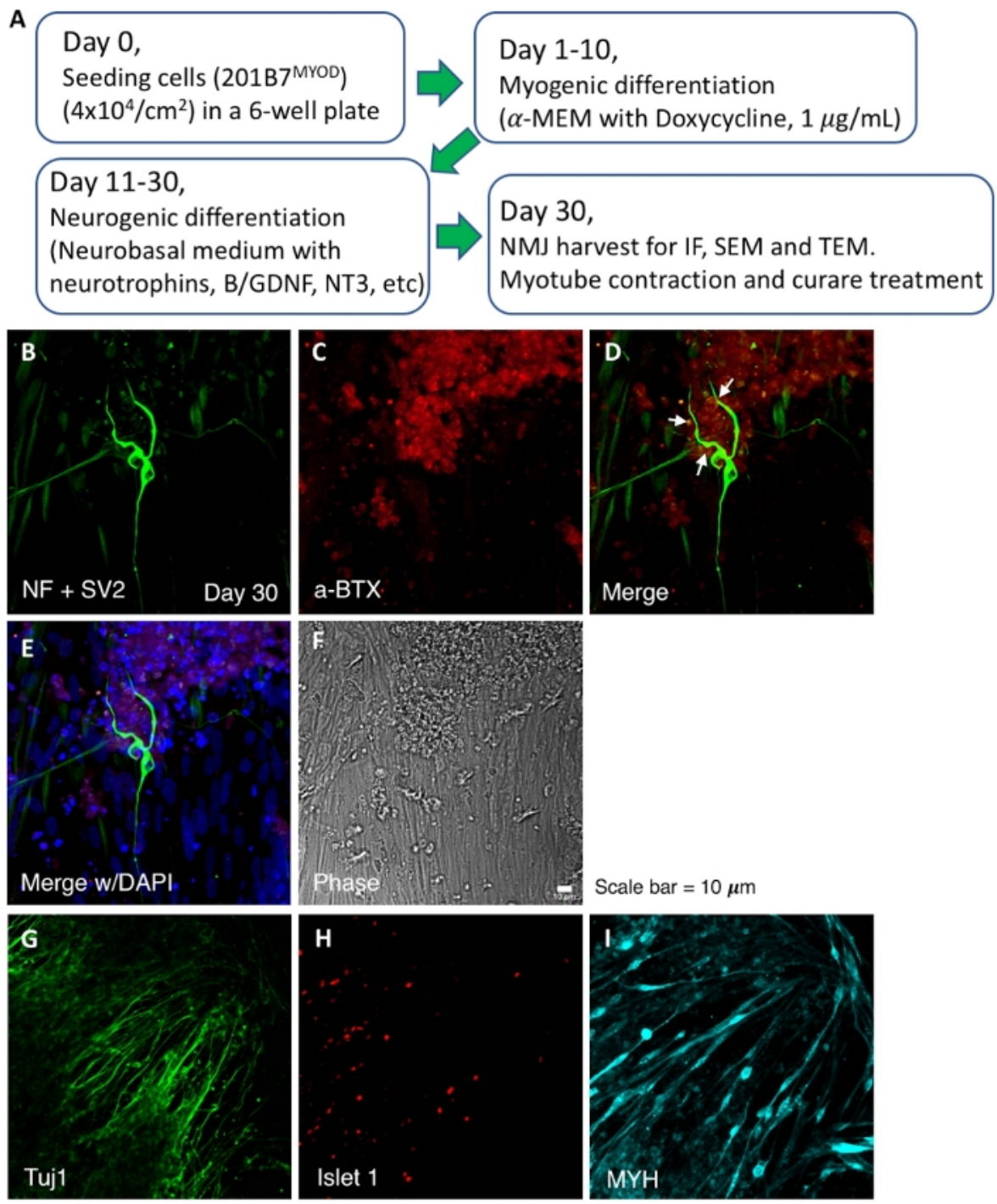

Scale bar $=10 \mu \mathrm{m}$
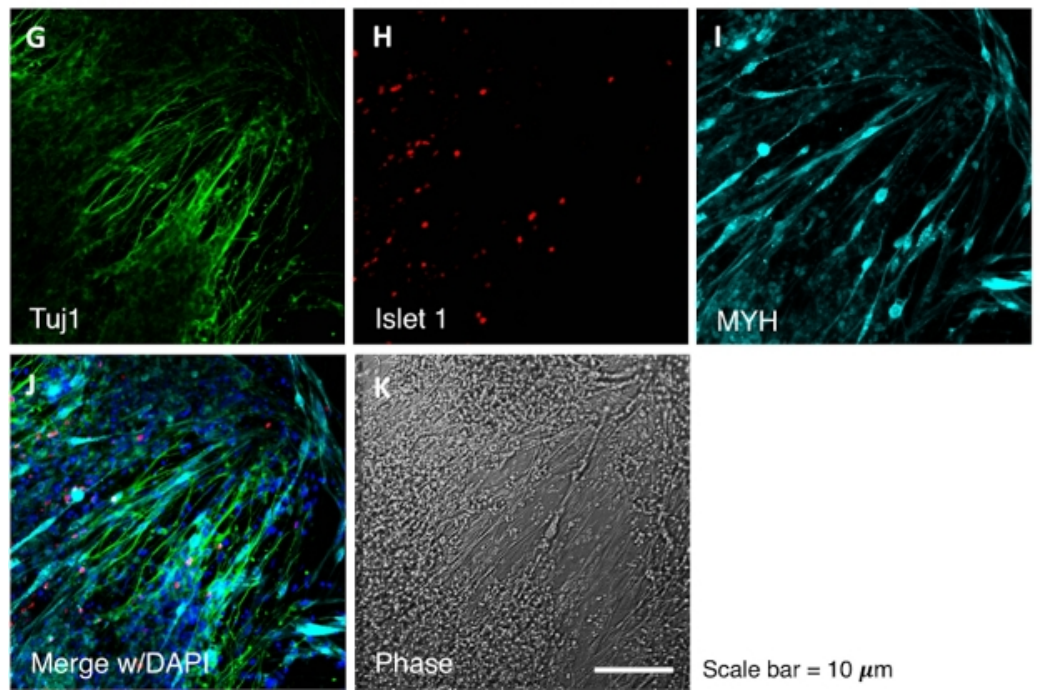

Figure 1: Flow chart for NMJ induction and IF images of NMJ culture. (A) A flow chart for the NMJ differentiation progression. (B-F) Detection of NMJ components, neurofilaments (NF), synaptic vesicles (SV2) and AChR. The white arrows in panel D indicate the NMJ. (G-K) Pre- and post-synaptic components of the NMJ. Tuj1 and Islet1 indicate the motor neuron, and myosin heavy chain (MYH) indicates the myotube. a-BTX, alpha-bungarotoxin. Please click here to view a larger version of this figure. 

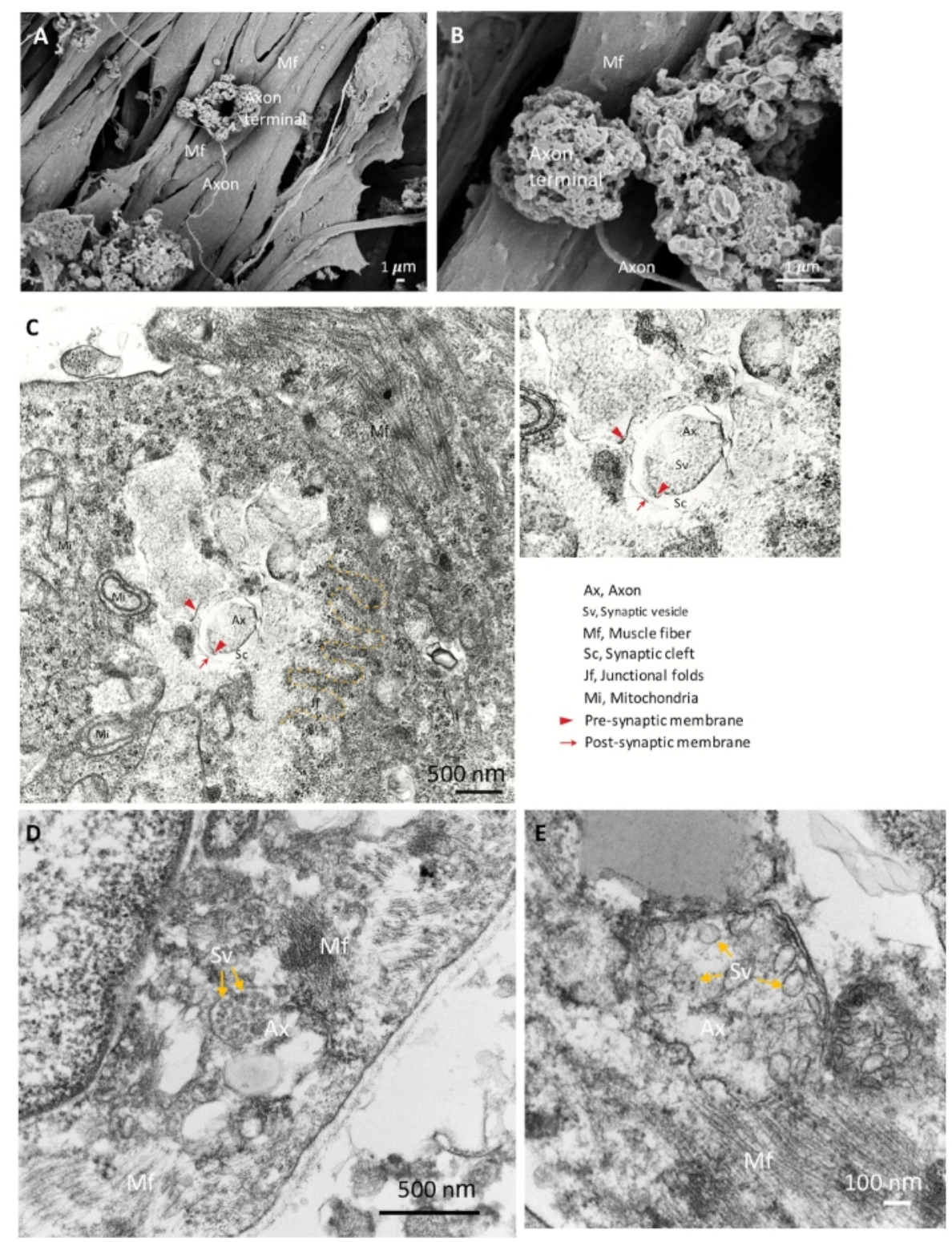

Figure 2: SEM and TEM images of mature NMJ. (A) The spatial distribution of NMJ components show the axon terminal anchored on the surface of the muscle fiber (Mf). (B) A higher magnification of the NMJ shows the axon terminals. (C) The ultrastructure of a matured NMJ with pre-synaptic axon terminal (red arrowheads) and synaptic vesicles (Sv), synaptic cleft (Sc) and post-synaptic parts (red arrows). Junctional folds (jf) are indicated by the yellow dashed line. Ax, axon. (D, E) The high magnification images show the synaptic vesicles in axon terminals (yellow arrows). Please click here to view a larger version of this figure. 


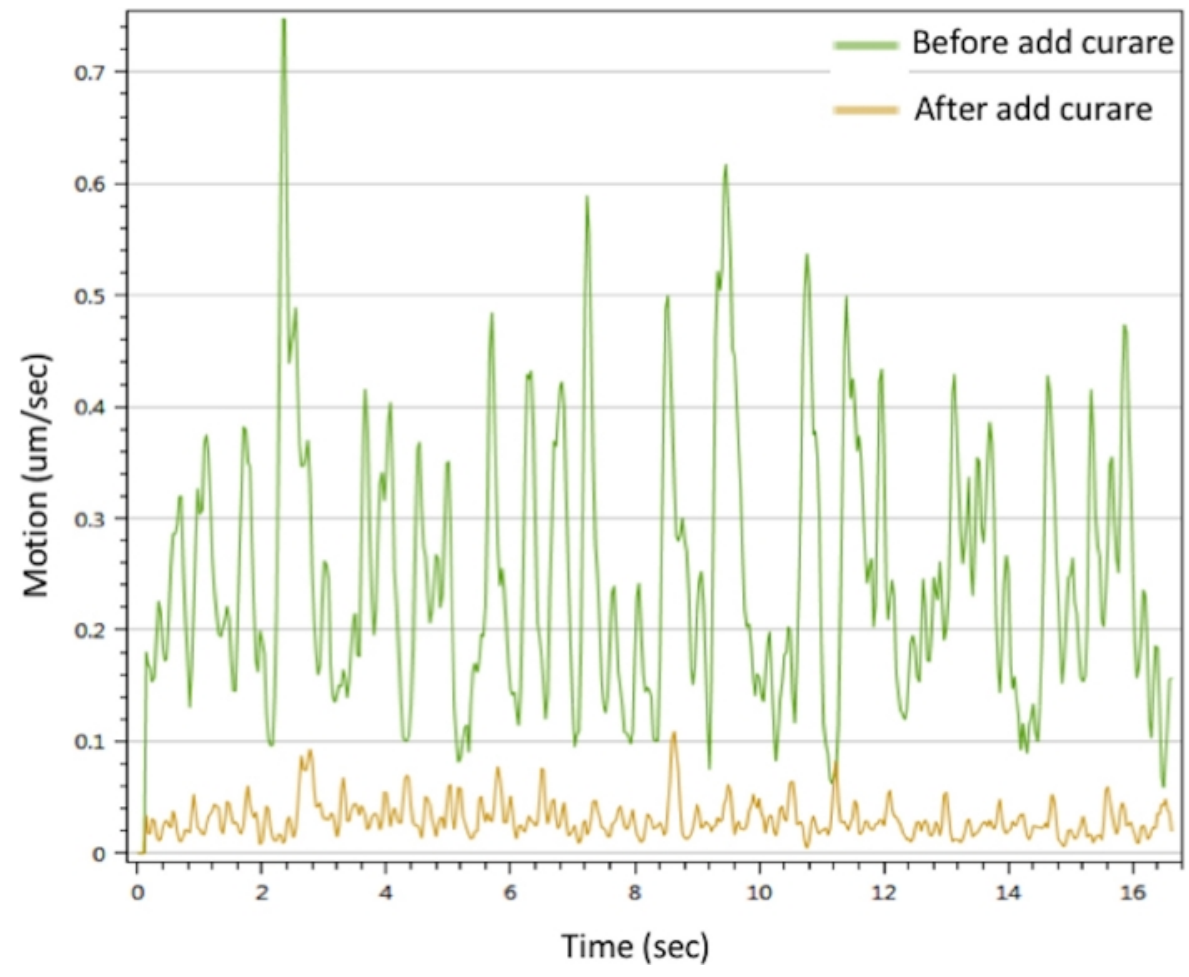

Figure 3: Myotubes contraction analysis of in vitro NMJ. Myotubes contraction were triggered with $25 \mathrm{mM} \mathrm{CaCl}_{2}$ solution (green line). The contractions were inhibited after treated with curare (yellow line). Please also see Supplementary Movie 1 and Supplementary Movie 2. Please click here to view a larger version of this figure.

Supplementary Movie 1: The myotubes contraction triggered by $\mathbf{C a}^{++}$. Please click here to download this video.

Supplementary Movie 2: The much weaker contraction of myotubes after curare treatment is shown. Please click here to download this video.

Supplementary Figure 1: The single staining images of neurofilaments (NF, white arrows) in NMJ culture.
The single staining images of synaptic vesicles (SV2, white arrows) in NMJ culture. Please click here to download this figure.

Supplementary Figure 2: The Schwann cells labeled by S-100 antibody in NMJ culture. Please click here to download this figure.

\begin{tabular}{|c|c|c|}
\hline $\begin{array}{c}\text { Myogenic differentiation } \\
\text { medium (MDM) }\end{array}$ & MEM-alpha & $500 \mathrm{~mL}$ \\
\hline & $100 \mathrm{mM} 2-\mathrm{ME}$ & $1117 \mu \mathrm{L}$ \\
\hline & Doxycycline & $1 \mu \mathrm{g} / \mathrm{mL}$ \\
\hline & KSR & $56 \mathrm{~mL}$ (final to $10 \%)$ \\
\hline
\end{tabular}




\begin{tabular}{|c|c|c|}
\hline & Pen/Strep & $2.5 \mathrm{~mL}$ \\
\hline 100mM 2-mercaptoethanol & total & $560 \mathrm{~mL}$ \\
\hline & 2-mercaptoethanol & $993 \mu \mathrm{L}$ \\
\hline NMJ medium & d.d. water & $1000 \mu \mathrm{L}$ \\
\hline & total & $500 \mathrm{~mL}$ \\
\hline & Neurobasal medium (NB) & $1 \times($ stock in $50 \mathrm{x})$ \\
\hline & B27 & $10 \mathrm{ng} / \mathrm{mL}$ \\
\hline & BDNF & $10 \mathrm{ng} / \mathrm{mL}$ \\
\hline & GDNF & $1 \times($ stock in 100x) \\
\hline & N2 & $10 \mathrm{ng} / \mathrm{mL}$ \\
\hline
\end{tabular}

Table 1: Formula of medium.

\section{Discussion}

Previous studies have reported methods for NMJ formation in vitro with sophisticated methods that require longtermed culture $8,10,12,13,20$. These achievements lead the progression of in vitro NMJ. However, the complicated methodologies hindered the access of NMJ studies. Our protocol avoids co-culture to generate NMJs efficiently and robustly in a single well. Three cell types in the NMJ were observed in our induction system, including the myotubes, the motor neurons and the Schwann cells ${ }^{18}$. In addition, mature morphology and function were verified.

Based on our previous study, the initial cell density is a critical factor for NMJ formation, and different cell densities induce various numbers of NMJs in the culture ${ }^{18}$. A low cell density $\left(<1 \times 10^{4} / \mathrm{cm}^{2}\right)$ induced a low number of neurons, which is unfavorable for NMJ formation. Although it would take much more time and resources to prepare a higher density of cells $\left(>1 \times 10^{5} / \mathrm{cm}^{2}\right)$, we recommend that the cell density be between $1.5 \times 10^{4} / \mathrm{cm}^{2}$ and $5 \times 10^{4} / \mathrm{cm}^{2}$ using our protocol. The NMJ generated by this protocol is a heterogeneous tissue with multiple cell types which is closer to mimicing physiological conditions. The myotubes are found to evenly distribute on a culture dish but the motor neurons appear randomly, of which the phenomenon results in the unevenly distribution of NMJs in a culture. This NMJ is suitable for qualitative studies instead of the analysis requiring homogeneous culture systems. We also used other cell lines, eq., H9 (ES cell line) ${ }^{18}$ and $A 11$ (iPS cell line, data not shown) to generate NMJ by this protocol. The NMJ can be generated successfully in morphology and function. The culture condition, nevertheless, needs to be optimized for different cell lines. 
The chemically triggered myotubes contraction and curaredependent inhibition confirmed that our in vitro NMJ was functional and could be potentially applied for practical assays. Spontaneous muscle contractions were observed randomly at 3-4 weeks of culture, but it could be avoided by extending the culture time to as long as two months ${ }^{18}$.

Various strategies have been published for generating in vitro NMJ of different maturation stages, but the in vitro NMJ generated in our system showed the significant maturity, as we can observe the transition of gamma to epsilon subunits of $\mathrm{AChR}$ during the culture ${ }^{18}$. Maturity is an important consideration for disease modeling with in vitro $\mathrm{NMJ}^{21}$. In conclusion, an in vitro NMJ with integrated structural components, maturity and function is of potential use for therapeutic development.

\section{Disclosures}

The authors have nothing to disclose.

\section{Acknowledgments}

We thank Dr. Sakurai for kindly providing 201B $7^{\mathrm{MYOD}}$. The electron microscopy study was supported by Keiko OkamotoFuruta and Haruyasu Kohda (Division of Electron Microscopic Study, Center for Anatomical Studies, Graduate School of Medicine, Kyoto University). The monoclonal antibody was developed by $\mathrm{HHMI} /$ Columbia University, obtained from the Developmental Studies Hybridoma Bank, created by the National Institute Child Health and Human Development of the $\mathrm{NIH}$, and maintained at the Department of Biology, University of lowa. We also thank Shiori Oshima, Kazuhiro Nakagawa and Eriko Matsui for motion vector analysis. This work was supported by funding from the Japan Society for the Promotion of Science KAKENHI, grant numbers 16H05352 and 20H03642 (to MKS); iPS Cell Research Fund (to CYL and MKS); Mochida Memorial Foundation for Medical and Pharmaceutical Research (to MKS); Takeda Science Foundation (to MKS); and a grant from The Program for Intractable Diseases Research utilizing Disease-specific iPS cells, which was aided by the Japan Agency for Medical Research and Development (17935400 to CYL and MKS, and 17935423 to MKS).

\section{References}

1. Hong, I. H. K., Etherington, S. J. Neuromuscular Junction. In: eLS. John Wiley \& Sons, Ltd. (2001).

2. Sanes, J. R., Lichtman, J. W. Development of the vertebrate neuromuscular junction. Annual Review of Neuroscience. 22, 389-442 (1999).

3. Jones, R. A. et al. Cellular and Molecular Anatomy of the Human Neuromuscular Junction. Cell Reports. 21 (9), 2348-2356 (2017).

4. Slater, C. R. The Structure of Human Neuromuscular Junctions: Some Unanswered Molecular Questions. International Journal of Molecular Sciences. 18 (10), 2183 (2017).

5. Comley, L. H., Nijssen, J., Frost-Nylen, J., Hedlund, E. Cross-disease comparison of amyotrophic lateral sclerosis and spinal muscular atrophy reveals conservation of selective vulnerability but differential neuromuscular junction pathology. Journal of Comparative Neurology. 524 (7), 1424-1442 (2016).

6. Shigemoto, K. et al. Muscle weakness and neuromuscular junctions in aging and disease. Geriatrics \& Gerontology International. 10, S137-S147 (2010).

7. Abicht, A., Müller, J., Lochmüller, H. Congenital Myasthenic Syndromes. GeneReviews. (2016). 
8. Faravelli, I. et al. Motor neuron derivation from human embryonic and induced pluripotent stem cells: experimental approaches and clinical perspectives. Stem Cell Research \& Therapy. 5 (4), 87-100 (2014).

9. Demestre, M. et al. Formation and characterisation of neuromuscular junctions between hiPSC derived motoneurons and myotubes. Stem Cell Research. 15 (2), 328-336 (2015).

10. Yoshida, M. et al. Modeling the early phenotype at the neuromuscular junction of spinal muscular atrophy using patient-derived iPSCs. Stem Cell Reports. 4 (4), 561-568 (2015).

11. Vilmont, V., Cadot, B., Ouanounou, G., Gomes, E. R. A system for studying mechanisms of neuromuscular junction development and maintenance. Development. 143 (13), 2464-2477 (2016).

12. Uzel, S. G. M. et al. Microfluidic device for the formation of optically excitable, three-dimensional, compartmentalized motor units. Science Advances. 2 (8), e1501429 (2016).

13. Santhanam, N. et al. Stem cell derived phenotypic human neuromuscular junction model for dose response evaluation of therapeutics. Biomaterials. 166, 64-78 (2018).

14. Davis, R. L., Weintraub, H., Lassar, A. B. Expression of a single transfected cDNA converts fibroblasts to myoblasts. Cell. 51 (6), 987-1000 (1987).

15. Tanaka, A. et al. Efficient and reproducible myogenic differentiation from human iPS cells: prospects for modeling Miyoshi Myopathy in vitro. PLoS One. 8 (4), e61540 (2013).
16. Furlan, A., Adameyko, I. Schwann cell precursor: a neural crest cell in disguise? Developmental Biology. 444, S25-S35, (2018).

17. Jessen, K. R., Mirsky, R. Schwann Cell Precursors; Multipotent Glial Cells in Embryonic Nerves. Frontiers in Molecular Neuroscience. 12 (69) (2019).

18. Lin, C. Y. et al. iPSC-derived functional human neuromuscular junctions model the pathophysiology of neuromuscular diseases. JCI Insight. 4 (18) (2019).

19. Reynolds, E. S. The use of lead citrate at high $\mathrm{pH}$ as an electron-opaque stain in electron microscopy. Journal of Cell Biology. 17 (1), 208-212 (1963).

20. Steinbeck, J. A. et al. Functional Connectivity under Optogenetic Control Allows Modeling of Human Neuromuscular Disease. Cell Stem Cell. 18 (1), 134-143 (2016).

21. Bucchia, M., Merwin, S. J., Re, D. B., Kariya, S. Limitations and Challenges in Modeling Diseases Involving Spinal Motor Neuron Degeneration in vitro. Frontiers in Cellular Neuroscience. 12, 61 (2018). 